\title{
Consideraciones Anátomo-Clínicas sobre el Nervio Radial en el Codo
}

\author{
Anatomical and Clinical Considerations about the Radial Nerve in the Elbow \\ Vicente Hugo Bertone; Nicolás Ernesto Ottone; Maximiliano Ariel Lo Tartaro; Nicolás García de Quirós; \\ Agustín Arrotea; Mario Dominguez; Diana Finkelstein; Paolo López Bonardi; Sergio Shinzato \& Carlos Medan
}

BERTONE, V. H.; OTTONE, N. E.; LO TARTARO, M. A.; GARCÍA DE QUIRÓS, N.; ARROTEA, A.; DOMINGUEZ, M.; FINKELSTEIN, D.; LÓPEZ BONARDI, P.; SHINZATO, S. \& MEDAN, C. Consideraciones anátomo-clínicas sobre el nervio radial en el codo. Int. J. Morphol., 26(2):437-444, 2008.

RESUMEN: El recorrido del nervio radial a través del codo constituye, para este elemento nervioso, un camino con riesgo de lesiones intrínsecas o extrínsecas. Cambios de la morfología de las estructuras osteomusculares que constituyen el desfiladero del nervio radial, tanto patológicos como traumáticos, pueden determinar el atrapamiento y compresión del mismo, determinando el daño del nervio y/o la inflamación localizada a nivel de las estructuras circundantes. Mediante la disección de 30 preparados, formolizados al 10\%, y el análisis subsecuente de la disposición de las estructuras musculares e inserción de las mismas, se determinaron los posibles puntos de riesgo anatómico que pudiesen alterar al nervio radial o a sus ramos en la canal bicipital lateral o en su ingreso y distribución en el parte proximal del antebrazo (relación con el músculo supinador y los músculos extensores radial largo y corto). Definimos 4 zonas de posible atrapamiento y compresión del nervio radial y sus ramos: 1. Septo intermuscular lateral. 2. Músculo extensor radial corto. 3. Músculo supinator, a nivel de la Arcada de Frohse. 4. Músculo supinator, a la salida de la masa muscular, en el dorso del antebrazo. La compresión del nervio radial a nivel del codo es una de las neuropatías del miembro superior, más frecuentes. El objetivo de este trabajo fue analizar las implicancias anatómicas del recorrido del nervio radial en su pasaje desde el brazo al antebrazo, especialmente las relaciones con las estructuras osteomusculares, cuyas alteraciones pueden ser las responsables de patologías compresivas del nervio radial que puedan llevar a dolor, parestesias, con o sin pérdida sensorial y/o impotencia funcional.

PALABRAS CLAVE: Nervio radial; Atrapamiento; Arcada de Frohse; Nervio interóseo antebraquial posterior.

\section{INTRODUCCIÓN}

El nervio radial deriva de la porción dorsal del plexo braquial y sus raíces constitutivas provienen prácticamente de todas las que lo forman, es decir, de las raíces cervicales $5^{\mathrm{a}}, 6^{\mathrm{a}}, 7^{\mathrm{a}}, 8^{\mathrm{a}} \mathrm{y}$, en proporción variable, de la $1^{\mathrm{a}}$ raíz torácica (Testut \& Latarjet, 1954; Bouchet \& Cuilleret, 1997). Luego de recorrer la axila y el surco del nervio radial del húmero, atraviesa el tabique intermuscular lateral del brazo, para ingresar en lo que denominamos el "desfiladero radial". Por lo tanto, se inicia a nivel de este tabique y finaliza en el borde inferior del fascículo superficial del músculo supinador, en el dorso del antebrazo.

El "desfiladero radial" consta de 3 porciones. La primera porción se extiende desde el sitio en que el nervio radial perfora el tabique intermuscular lateral del brazo, hasta el nivel de la interlínea de la articulación húmero-radial del codo. En esta porción el nervio radial proporciona los ramos nerviosos destinados a los músculos con origen en el epicóndileo lateral del húmero, que conforman el límite anterolateral del desfiladero: músculos braquioradial y extensor radial largo del carpo (MERLC), extensor radial corto del carpo (MERCC), dispuestos sucesivamente de proximal a distal. Medialmente son los músculos braquial y bíceps braquial los que delimitan esta primera porción.

La segunda porción se extiende desde la interlínea articular del codo hasta el borde superior del haz superficial del músculo supinador. Este borde recibe el nombre de "Arcada de Frohse" (AF), debido a que fue descrito por primera vez por Frohse \& Fränkel en 1908 (Spinner, 1968). Es en esta porción donde el nervio radial, entre 1,5 y 2,5 cm por debajo de la interlínea articular del codo, se divide en sus 
dos ramos terminales: superficial superficial (anterior y sensitivo) y un ramo profundo (posterior y motor), el cual después de inervar la músculatura extensora más proximal pasa a denominarse nervio interóseo antebraquialposterior (NIAP). (Bianchi, 2002)

Finalmente, la tercera porción se extiende desde la AF hasta el borde inferior del músculo supinador. El NIAP corre por debajo de la AF, y penetra en un túnel carnoso espiroidal, verdadero "estuche protector" (Roust \& Espeche, 1969), formado por los haces superficial y profundo del músculo supinador (Spinner; Roust \& Espeche; Ferreira et al., 1984; Lafalla, 1987; Debouck \& Rooze, 1995; Riffaud et al., 1999; Ozturk et al., 2005). El NIAP abandona este túnel carnoso a través del borde inferior del músculo supinador, el que le permite ingresar al compartimiento posterior del antebrazo, donde inmediatamente se divide en sus ramas terminales de inervación de los músculos del sector.

La neuropatía por compresión del nervio radial a nivel del codo es muy frecuente (Leppilathi et al., 2001). El recorrido de este elemento nervioso a través del "desfiladero radial" constituye un camino con riesgo de lesiones intrínsecas o extrínsecas.

Runge, en 1873, fue uno de los primeros en describir el "codo de tenista" (Leppilathi et al.). Guillain \& Courtellemont (en Debouck \& Rooze) también postularon su hipótesis sobre la compresión del nervio radial por parte del músculo supinador, en 1905. Koppel \& Thompson (1963) reportaron el primer caso de atrapamiento del NIAP por la AF. Capener (1966) describió la vulnerabilidad del NIAP a nivel de la AF. Spinner estatificó la morfología de la AF y la compresión del NIP por la AF, en 1968 (Ozturk et al.). Ozturk et al. desarrollaron una técnica de medición de la AF, que es utilizada en este trabajo para determinar la morfología de la misma.

El objetivo de este trabajo consistió en determinar la distribución del nervio radial en el "desfiladero", cuáles son las estructuras que pueden determinar el atrapamiento y compresión del nervio radial, y cómo se verá implicado en dicha patología compresiva, que puede llevar a dolor, parestesias, con o sin pérdida sensorial y/o impotencia funcional y que se encuentran directamente relacionadas con alteraciones en la vida social y laboral de las personas afectadas.

\section{MATERIAL Y MÉTODO}

Mediante la disección de 30 preparados de brazo y antebrazo (18 derechos y 12 izquierdos), formolizados al $10 \%$, fueron analizados particularmente la anatomía de la región del codo, la distribución del nervio radial y los puntos de riesgo anatómico que pudieren alterar al elemento nervioso en su ingreso y distribución en el "desfiladero radial" (relación con el músculo supinador, MERLC y MERCC).

Para analizar la AF, se siguieron algunos de los lineamientos planteados por Ozturk et al. Este autor consideró los siguientes datos para medir la AF:

\section{- SL: extremo superolateral de la AF}

- IM: extremo inferomedial de la AF

- L: longitud de la AF.

- A: Ancho de la AF.

- Distancia AF: distancia a la que se encuentra la AF de la interlínea articular del codo.

- Longitud del antebrazo

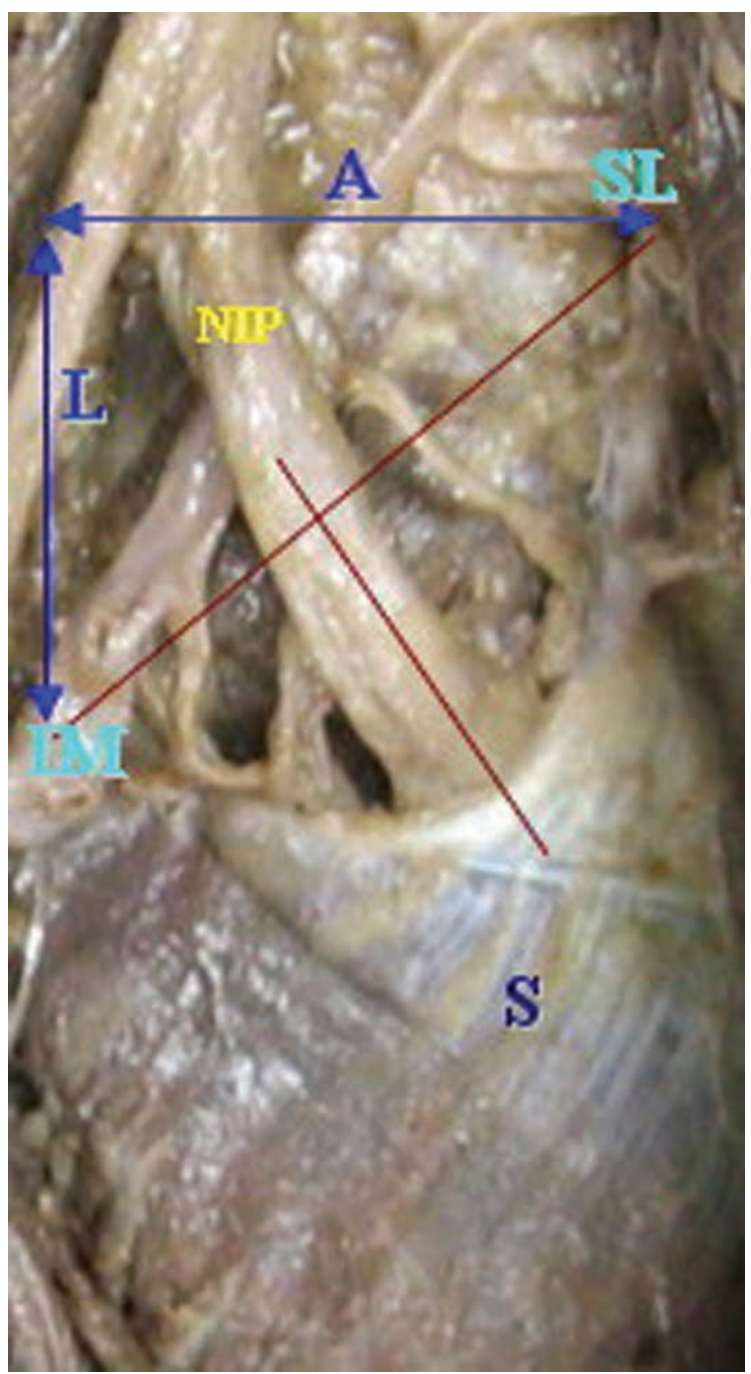

Fig. 1. Método de medición de la Arcada de Frohse (AF), desarrollado por Ozturk et al., 2005. S. Músculo supinador; Extremo inferomedial de la AF; SL. Extremo superolateral de la AF; Longitud de la AF; A. Ancho de la AF; NIP. Nervio interóseo antebraquial posterior. 
BERTONE, V. H.; OTTONE, N. E.; LO TARTARO, M. A.; GARCÍA DE QUIRÓS, N.; ARROTEA, A.; DOMINGUEZ, M.; FINKELSTEIN, D.; LÓPEZ BONARDI, P.; SHINZATO, S. \& MEDAN, C. Consideraciones anátomo-clínicas sobre el nervio radial en el codo. Int. J. Morphol., 26(2):437-444, 2008.

\section{RESULTADOS}

La distribución de los ramos del nervio radial en el "desfiladero" se encuentran expresados en la Tabla I. El nervio radial se divide por debajo de la interlínea articular del codo, a una distancia de entre 1,5 y $2,5 \mathrm{~cm}$.

El músculo braquioradial en un caso, se originaba bastante proximalmente, cerca de la inserción de los músculos deltoides y braquial. En el resto de las preparaciones no fueron encontradas variaciones anatómicas considerables con respecto a la inserción del músculo braquioradial.

La AF corresponde al borde superior del haz superficial del músculo supinador (Figs. 2-5). Presenta una forma semicircular, de concavidad superior. La AF se presentó en el 30\% de tipo "tendinomuscular" (Fig. 4) y en el 53,3\% de tipo "tendinosa" (Fig. 5).

Tabla I. Ramos nerviosos para los músculos braquioradial extensores radiales largo y corto del carpo y supinador emitidos a nivel del codo.

\begin{tabular}{|c|c|c|c|c|}
\hline \multirow{2}{*}{ Músculo } & \multicolumn{3}{|c|}{$\mathrm{N}^{\mathrm{o}}$ de ramos } & \multirow{2}{*}{ Tronco común } \\
\hline & 1 & 2 & 3 & \\
\hline Braquioradial & $36,6 \%(11)$ & $30,0 \%(9)$ & $6,7 \%(2)$ & $26,7 \% *(8)$ \\
\hline $\begin{array}{l}\text { Extensor radial } \\
\text { largo del carpo }\end{array}$ & $53,33 \%(16)$ & $16,7 \%(5)$ & $3,33 \%(1)$ & $26,7 \% *(8)$ \\
\hline $\begin{array}{l}\text { Extensor radial } \\
\text { corto del carpo }\end{array}$ & $100 \%(30)$ & - & - & - \\
\hline Supinador & $83,3 \%$ & $16,7 \%(5)$ & - & - \\
\hline
\end{tabular}

Los músculos braquioradial y extensor radial largo del carpo son inervados directamente por el nervio radial. Los músculos extensor radial corto del carpo y supinador son inervados por el ramo profundo del nervio radial.
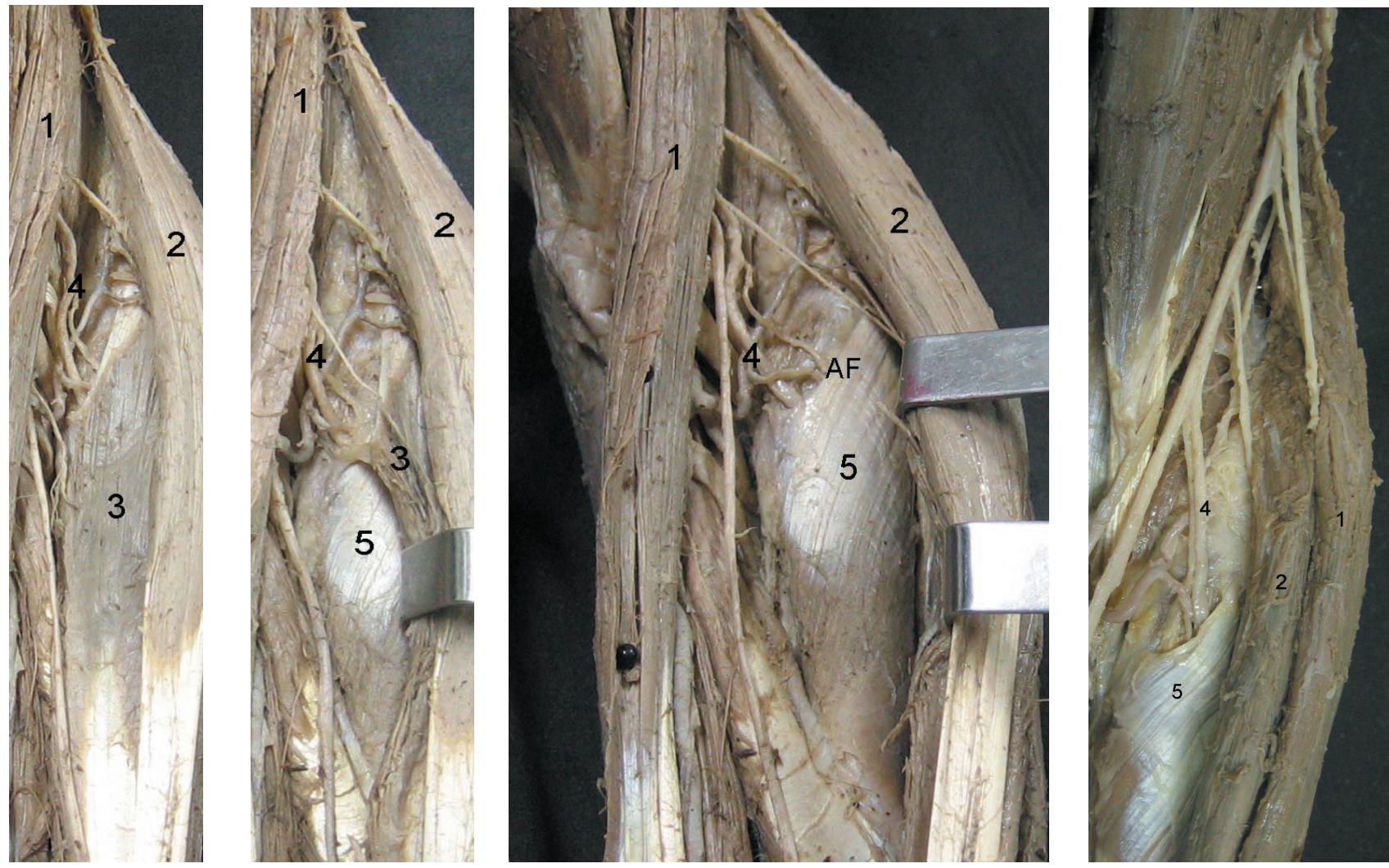

Fig. 2. Plano correspondiente al músculo extensor radial corto del carpo. Fig. 3. El músculo extensor radial corto del carpo intercambia fibras de asociación con el músculo supinador, al cual se fusiona, conformando de esta manera una segunda arcada al nervio interóseo posterior. Fig. 4. Observación de la Arcada de Frohse de tipo "tendinomuscular". Fig. 5. Arcada de Frohse de tipo "tendinosa". 1. Músculo braquiorradial; 2. Músculo extensor radial largo del carpo; 3. Músculo extensor radial corto del carpo; 4. Nervio interóseo antebraquial posterior; 5. Músculo supinador; AF. Arcada de Frohse. 


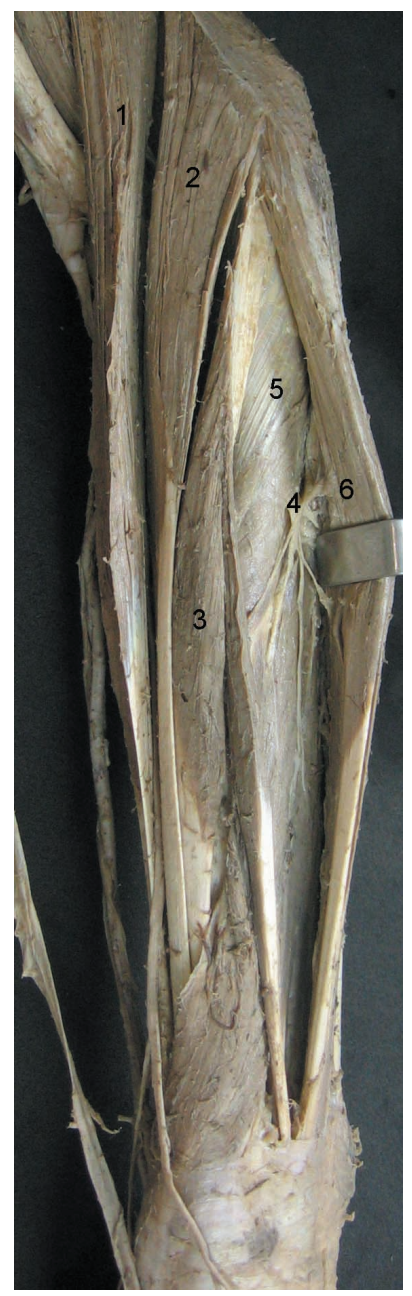

Fig. 6. Región antebraquial posterior, se aprecia al nervio interóseo posterior emergiendo en esta región y distribuyendo sus ramos musculares, a través del borde inferior del músculo supinador.

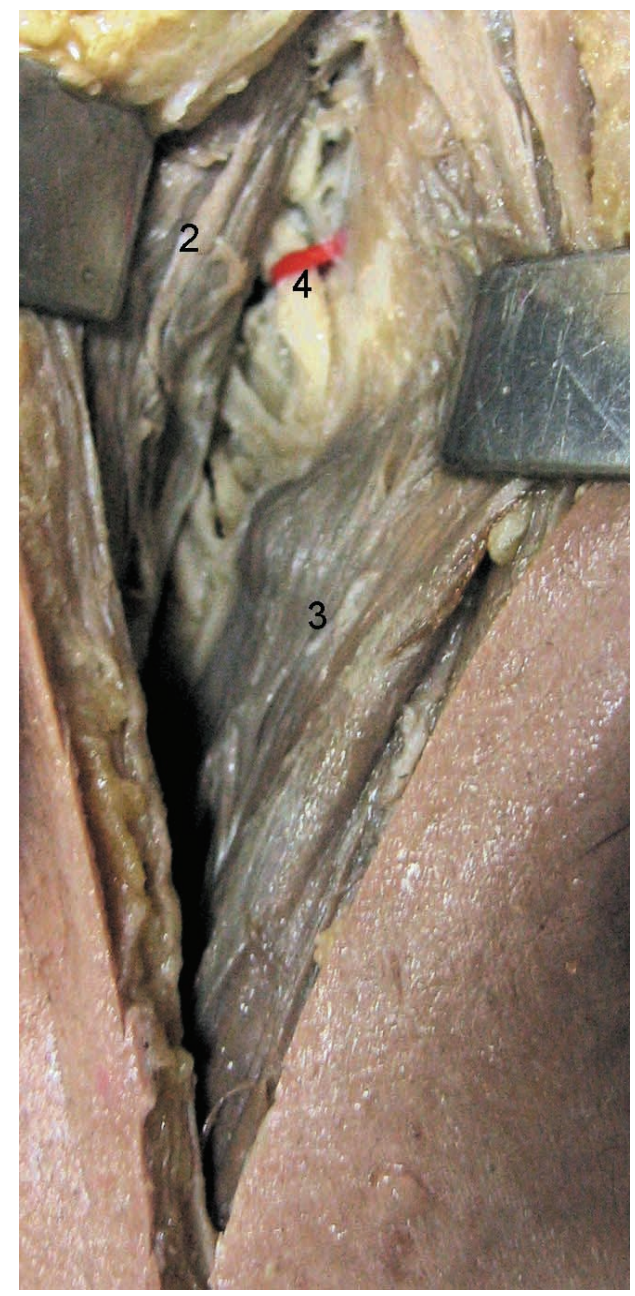

Fig. 7. Vía de acceso A. Entre los músculos extensores radiales largo y corto del carpo al plano de éste último músculo. A este nivel se observa el nervio interóseo posterior.

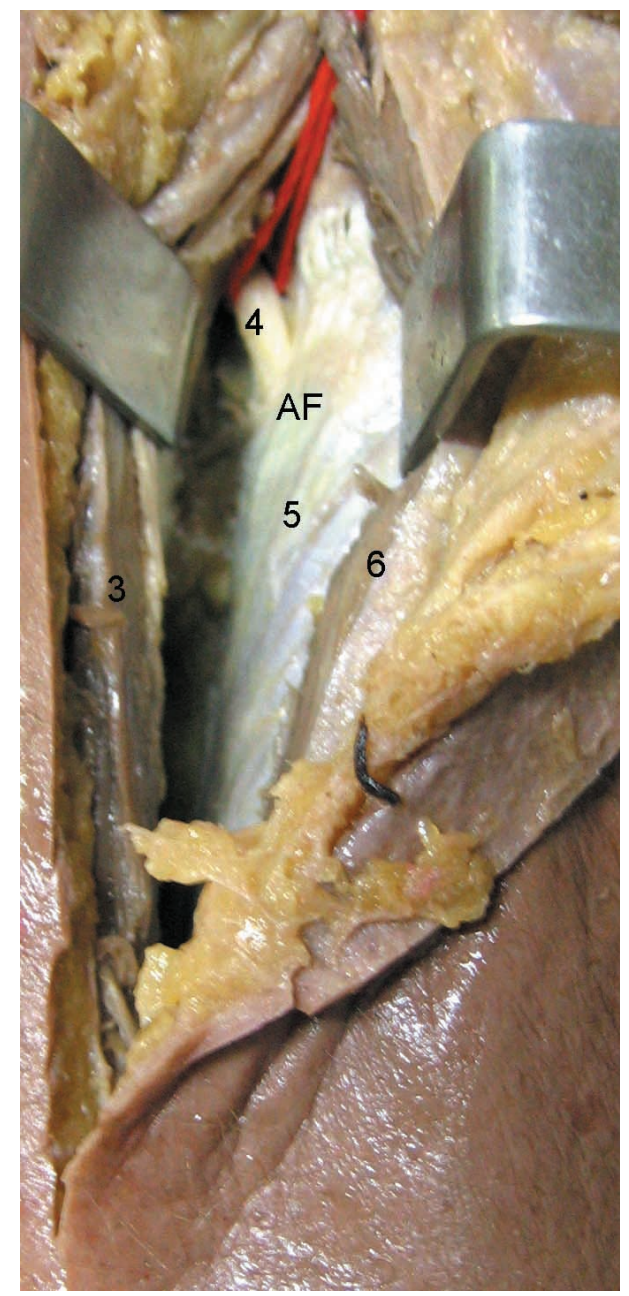

Fig. 8. Vía de acceso B. Se accede a la Arcada de Frohse en forma directa, a través de la interlínea que separa a los músculos extensor radial corto del carpo y extensor de los dedos.

1. Músculo braquiorradial; 2. Músculo extensor radial largo del carpo; 3. Músculo extensor radial corto del carpo; 4. Nervio interóseo antebraquial posterior; 5. Músculo supinador; 6. Músculo extensor de los dedos; AF. Arcada de Frohse.

ancho de la AF, fue de $14,4 \mathrm{~mm}$ y la longitud de $12,7 \mathrm{~mm}$. La distancia de la AF con respecto a la interlínea articular del codo fue de $37,4 \mathrm{~mm}$.

Describimos dos posibles accesos quirúrgicos que permiten descubrir la Arcada de Frohse logrando una exposición exacta de la misma. Colocando el antebrazo en pronación completa y, tomando como punto de partida el epicóndilo lateral del húmero, realizamos los siguientes procedimientos:

- El primer acceso (A: a-a') corresponde a la exposición del límite de separación de los músculos MERLC y MERCC.
La incisión se traza desde el epicóndilo lateral del húmero siguiendo el borde lateral del antebrazo (alrededor de $8 \mathrm{~cm}$ ). Este acceso permite ingresar al plano del MERCC, el cual puede estar conformando por una arcada en conjunción a la AF, y allí, ubicar al NIAP. (Fig. 7).

- El segundo acceso (B: b-b'), permite llegar directamente al músculo supinador, y por tanto, a la AF. Se logra trazando una incisión que, parte del epicóndilo lateral del húmero y sigue el trayecto de la interlínea de separación del MERCC y músculo extensor de los dedos (MED) (también de $8 \mathrm{~cm}$ ), cuya separación permite encontrar al músculo supinator, y de esa manera, descubrir la AF. (Fig. 8). 
BERTONE, V. H.; OTTONE, N. E.; LO TARTARO, M. A.; GARCÍA DE QUIRÓS, N.; ARROTEA, A.; DOMINGUEZ, M.; FINKELSTEIN, D.; LÓPEZ BONARDI, P.; SHINZATO, S. \& MEDAN, C. Consideraciones anátomo-clínicas sobre el nervio radial en el codo. Int. J. Morphol., 26(2):437-444, 2008.

\section{DISCUSIÓN}

El nervio radial recorre el "desfiladero radial", pero debe estar preparado para las eventuales elongaciones que pudiese sufrir en los movimientos de pronación-supinación. Como relatan Roust \& Espeche, es característico el trayecto del nervio radial en doble curva de sentido inverso (curva proximal de sentido postero-anterior sobre el surco del nervio radial y la curva distal ubicada como un "emparedado" en el espesor de un túnel carnoso dentro del músculo supinador, de sentido inverso antero-posterior).

El ancho de la AF, fue de $14,4 \mathrm{~mm}$ y la longitud de $12,7 \mathrm{~mm}$, mientras que para Ozturk et al. las mismas fueron de 10,1 y $8,6 \mathrm{~mm}$, respectivamente. La distancia de la AF con respecto a la interlínea articular del codo fue de $37,4 \mathrm{~mm}$, mientras que para Ozturk etal. fue de 46,2 mm. Quizás estas pequñas diferencias puedan deberse a variantes relativas a la población considerada (argentinos/ turcos) como así también a la casuística considerada (30 casos/55 casos) (Ozturk et al.).

Observamos algunas diferencias morfológicas en la AF con respecto a los estudios de Ozturk et al. Sin embargo, nuestros resultados son similares con los alcanzados por Debouck \& Rooze. En cambio, Spinner señaló que en el 30\% de los casos la AF es tendinosa, porcentaje bastante inferior al $87 \%$ de Ozturk et al., el 64,1\% de Debouck \& Rooze y al 53,3\% de nuestra muestra.

El MERCC y MERLC en los mamíferos inferiores están representados por un solo músculo, sin embargo, hay casos descritos en el hombre; al igual que Bergman et al., no encontramos fusión muscular. En un caso se encontró que los músculos estaban más desarrollados de lo normal, en comparación con el resto de los preparados, debido a que se trataba de un individuo que por su morfología anatómica, habría realizado actividades laborales de gran esfuerzo físico. Más allá de esto, no se encontraron variaciones anatómicas. Es importante considerar la disposición del MERCC, en cuanto a su origen. Por su borde medial, es capaz de formar una especie de arcada al NIAP, de conformación tendino-muscular, la cual toma inserción e intercambia fibras de asociación con la AF.

Hay autores que describen la existencia de un músculo braquiradial accesorio, denominado por Lauth músculo supinador largo accesorio o músculo braquioradial corto menor; éste se presenta adyacente al músculo braquioradial, insertándose en la tuberosidad radial y actuando como un verdadero supinador (Bergman et al., 2006).
Para el músculo supinador, consideramos importante el conocimiento anatómico de las puertas de entrada y salida del túnel carnoso espiroidal, que determinan los fascículos superficial y profundo del músculo y que permiten el pasaje del nervio radial del compartimiento anterior al posterior del antebrazo. La entrada: AF es considerada como el principal sitio de atrapamiento y compresión del NIAP en la patología del codo. La emergencia es el borde inferior de la masa superficial del músculo supinador, la cual permite el acceso del nervio al compratimiento posterior del antebrazo.

Definimos cuatro entidades anatómicas de posible atrapamiento y compresión: 1. tabique intermuscular lateral del brazo (n. radial); 2. borde medial del MERCC (NIP); 3Arcada de Frohse (NIP); 4. borde inferior de la masa superficial del músculo supinador (NIAP).

El principal sitio de atrapamiento y compresión del nervio radial es la AF, donde el NIAP se ve comprometido (Spinner; Roust \& Espeche; González, 1984; Lafalla, 1980; Debouck \& Rooze; Kirici \& Irmak, 2004; Ozturk et al.). El nervio radial es, principalmente, un nervio motor, por lo que la presencia de debilidad motora con extensión de los dedos o el pulgar y dolor en la parte lateral del codo, debe hacer sospechar de atrapamiento y compresión del NIAP (Mazurek \& Shin, 2001).

Pero si bien la AF es el principal sitio de atrapamiento, esto no significa que su existencia sea variable. Todo lo contrario, la AF es una estructura semicircular anatómica normal (presente en el 100\% de los casos) (Spinner; Roust \& Espeche; González; Debouck \& Rooze; Kirici \& Irmak; Ozturk et al.), que presenta variaciones en cuanto a su morfología. Estas variaciones son la base de la patología compresiva del NIAP.

A partir de nuestra investigación fue posible establecer que la AF es de tipo tendinosa en el 53,3\% de los casos (30\% Spinner; 64,1\% Debouck \& Rooze; 57\% Kirici \& Irmak; $87 \%$ Ozturk et al.). Clínicamente, esta AF tendinosa, es la que determinaría con mayores probabilidades, el desarrollo del síndrome del nervio interóseo antebraquial posterior (Spinner; Roust \& Espeche; Debouck \& Rooze; Kirici \& Irmak; Ozturk et al.), ya que en estos casos posee una densidad aumentada con un hiato estrecho, los que constituyen los factores potenciales de desarrollo de atrapamiento y compresión del nervio interóseo antebraquial posterior.

Frohse \& Fränkel, en 1908, fueron los primeros en describir esta estructura como normal, y Spinner en su investigación en 25 cadáveres adultos y 10 fetos, estableció 
que ninguno de los fetos presentaba una AF tendinosa, y que por lo contrario, la ésta era de tipo muscular. Lo que se puede suponer es que la conversión de dicha arcada en una estructura tendinosa pueda deberse a los movimientos de rotación repetitivos que los individuos realizan para llevar a cabo distintas actividades (Spinner; Ferreira et al., Debouck \& Rooze et al.; Kirici \& Irmak; Ozturk et al.).

Consideramos que la segunda zona en importancia de atrapamiento del NIAP lo constituye el borde medial del MERCC. Éste presenta una disposición tendino-muscular, que le forma al NIAP una especie de arcada, inmediatamente por delante de la clásica de Frohse (Younge \& Moise, 1994; Debouck \& Rooze; Riffaud et al.; Ozturk et al.).

Con respecto al borde inferior de la masa superficial del músculo supinador, sitio por donde emerge el NIAP para ubicarse en el dorso del antebrazo e inervar a todos los músculos del sector, es la última zona de posible atrapamiento y compresión del nervio en el desfiladero del radial (Leppilathi et al.; Kirici \& Irmak).

Es necesario considerar que la compresión del NIAP en la AF no sólo se debe a su formación tendinosa, sino que a la eventual existencia de una condición patológica a ese nivel, como la presencia de un linfonodo, lipoma, etc. (Bowen \& Stone, 1966; Sharrard, 1966; Werner, 1987; Monteiro et al., 2002).

Durante la realización de trabajo pesado (obreros de la construcción, por ejemplo) como en deportistas (tenistas, especialmente), el movimiento de rotación repetitivo del antebrazo (extensión y prono-supinación) y la posibilidad de hipertrofia de los músculos supinador y MERCC, aumentan la consistencia de la AF, del borde medial del MERCC, y también posiblemente del borde distal del músculo supinador, provocando compresión del nervio, isquemia del mismo y desarrollo del síndrome del NIAP (Sharrard; Roles \& Maudsley, 1972; Jalovaara \& Lindholm, 1989).

Hay autores como Riffaud et al., que consideran que la arcada vascular que le forma la arteria recurrente radial anterior y sus ramas al NIAP, a nivel de la AF, puede provocar compresión del nervio. Younge \& Moise, no coinciden con dicha apreciación. Nosotros no encontramos determinantes específicos de dicha posibilidad.

Un aporte interesante realizaron Woltman \& Learmonth (en Debouck \& Rooze), quienes describieron un caso de parálisis del NIAP donde el nervio no pasaba entre las dos porciones del músculo supinador sino que seguía un trayecto superficial, entre las fascias del músculos supinador y del músculo extensor de los dedos. No encontramos casos de este tipo.
Con respecto a la distribución del nervio radial, la principal diferencia entre nuestra investigación y el trabajo de Ferreira et al. radica en la existencia de un tronco común que proporciona los ramos nerviosos destinados a los músculos braquioradial y MERLC. Nosotros encontramos este tronco común en el 26,7\% de los casos, mientras que Ferreira et al. no hicieron hallazgos de este tipo. Por su parte, estos mismos autores describen una mayor presencia de un ramo directo para el músculo braquiradial $(63,6 \%)$, comparado con el $36,6 \%$ hallado en nuestra muestra.

Al igual que en la descripción realizada por los anatomistas clásicos, el MERCC presentó un único ramo de inervación, de calibre mayor en comparación a los ramos destinados al resto de los músculos (Ferreira et al.; Riffaud et al.). Con respecto al origen de los ramos destinados al músculo supinador, éstos lo hacen siempre inmediatamente por encima de la AF, desprendiéndose del NIP, salvo en un caso, el cual va a ser provisto por la ramo terminal superficial del nervio radial (Ferreira et al.).

Para acceder a la AF y desarrollar la descompresión del NIAP, hay autores que eligen la exposición de los músculos braquiradial MERLC. Sin embargo, esta técnica presenta la desventaja de permitir una limitada exposición de la arcada. Nosotros describimos dos vías de acceso que consideramos las indicadas para visualizar en forma directa la arcada y poder llevar a cabo de manera segura la descompresión del NIAP (Capener; Riffaud et al.). Entre la dos vías de acceso, elegimos aquella que nos sitúa en la interlínea de separación el MERCC y el músculo extensor de los dedos (Capener; Witt \& Kaminemi), la cual permite el descubrimiento del músculo supinador, y el acceso directo a la AF.

Consideramos que la AF es una estructura anatómica normal y la definimos como el principal sitio de atrapamiento y compresión nerviosa. A su vez, es el NIAP, el asiento de lesiones traumáticas, inflamatorias u otras, por compresión a este nivel. Los factores potenciales de trauma corresponden a la morfología tendinosa de la AF, a su hiato de ingreso estrecho, y también a la posibilidad de hipertrofia del músculo supinador debido a actividades de tipo laboral o deportivo. La presencia de otro tipo de factores como lipoma, linfonodo, etc. se da con menor frecuencia.

El conocimiento de la anatomía de la AF, el modo de distribución del nervio radial en su desfiladero, y las distintas vías de acceso para el hallazgo de la arcada, son de fundamental importancia para el correcto diagnóstico, tratamiento y recuperación del miembro superior afectado por la patología compresiva del nervio interóseo antebraquial posterior. 
BERTONE, V. H.; OTTONE, N. E.; LO TARTARO, M. A.; GARCÍA DE QUIRÓS, N.; ARROTEA, A.; DOMINGUEZ, M.; FINKELSTEIN, D.; LÓPEZ BONARDI, P.; SHINZATO, S. \& MEDAN, C. Consideraciones anátomo-clínicas sobre el nervio radial en el codo. Int. J. Morphol., 26(2):437-444, 2008.

BERTONE, V. H.; OTTONE, N. E.; LO TARTARO, M. A.; GARCÍA DE QUIRÓS, N.; ARROTEA, A.; DOMINGUEZ, M.; FINKELSTEIN, D.; LÓPEZ BONARDI, P.; SHINZATO, S. \& MEDAN, C. Anatomical and clinical considerations about the radial nerve in the elbow. Int. J. Morphol., 26(2):437-444, 2008.

SUMMARY: The radial nerve route through elbow constitutes, for this nervous element, a way with risk of intrinsic or extrinsic injuries. Morphologic changes of the structures that constitute the defile of the radial nerve, pathological as much traumatic, can determine it atrapment and compression, determining the damage of the nerve and/or the inflammation located at level of the surrounding structures. 30 forearm-elbow, preserved with formol to $10 \%$, were dissected, and the subsequent analysis of the disposition of the muscular structures and insertion of the same ones, will determine the possible points of anatomical risk that they will affect the radial nerve or its branches in the brachial track or in the proximal forearm (relation with the supinator muscle and extensor carpi radialis longus and brevis muscles). We defined 4 zones of atrapment and compression of the radial nerve and its branches: 1 . External intermuscular setum; 2. Extensor carpi radialis brevis muscle; 3. Supinator muscle, at level of the Frohse's Arch; 4. Supinator muscle, when coming out of the muscular mass, in the back of the forearm. The compression of the radial nerve at level of the elbow is one of the frequent neuropathies of the superior member. It is for that reason that the objective of this work consists of analyzing the anatomical aspects of the route of the radial nerve in its passage from the arm to the forearm, specially relations with morphological structures, whose alterations determines pathologies of the radial nerve which can take to pain, parestesies, with lost sensorial and functional impotence.

KEY WORDS: Radial nerve; Atrapment; Frohse's Arch; Posterior interosseous nerve.

\section{REFERENCIAS BIBLIOGRÁFICAS}

Bergman, R. A.; Afifi, A. K. \& Miyauchi, R. Ilustrated encyclopedia of human anatomic variation "Opera". January 2006. http://www.anatomyatlases.org/ AnatomicVariants/AnatomyHP.shtml.

Bianchi, F. H. Ramification of the superficial branch of the radial nervus. Rev. Chil. Anat., 20(3):247-50, 2002.

Bouchet, A. \& Cuilleret, J. Anatomía: descriptiva, topográfica y funcional. Miembro Superior. $1^{\mathrm{a}}$ ed. Buenos Aires, Médica Panamericana, 1997.

Bowen, T. L. \& Stone, K. H. Posterior interosseous nerve paralysis caused by a ganglion at the elbow. J. Bone Joint Surg. Br., 48(4):774-6, 1966.

Capener, N. The vulnerability of the posterior interosseous nerve of the forearm: a case report and an anatomical study. J. Bone Joint Surg. Br., 48(4):770-3, 1966.

Debouck, C. \& Rooze, M. The arcade of Fröhse: an anatomic study. Surg. Radiol. Anat., 17:245-8, 1995.

Ferreira, L. N.; Dommaco, S. M.; Lerner, F. \& Silberman, A. Inervación del epicóndilo y de los músculos epicondíleos. II Congreso de la Sociedad Rioplatense de Anatomía. Punta del Este, 1984.

Gonzalez R. E. La arcada de Frohse y sus relaciones neurovasculares. XXI Congreso de la Sociedad Rioplatense de Anatomía, 1984.
Jalovaara, P. \& Lindholm, R.V. Decompression of the posterior interosseous nerve for tennis elbow. Arch. Orthop. Trauma Surg., 108:243-5, 1989.

Kirici, Y. \& Irmak, M. K. Investigation of two possible compression sites of the deep branch of the radial nerve and nerve supply of the extensor carpi radialis brevis muscle. Neurol. Med. Chir. (Tokyo), 44:14-9, 2004.

Koppel, H. P. \& Thompson, W. A. L. Peripheral entrapment neuropathies. Baltimore, Williams and Wilkins, 1963. pp.131-42.

Lafalla, R. M. Relaciones de la rama posterior del nervio radial con la cabeza del radio e inervación motora de los músculos posteriores del antebrazo. XVII Congreso de la Sociedad Rioplatense de Anatomía, 1980.

Lafalla, R. M. Relaciones de la rama posterior del nervio radial con la cabeza del radio e inervación motora de los músculos posteriores del antebrazo. Mayor casuística. XXIV Congreso de la Sociedad Rioplatense de Anatomía, 1987.

Leppilahti, J.; Raatikainen, T.; Pienimäki, T.; Hänninen, A. \& Jalovaara P. Surgical treatment of resistant tennis elbow. A prospective, randomised study comparing decompression of the posterior interosseous nerve and lengthening of the tendon of the extensor carpi radialis brevis muscle. Arch. Orthop. Trauma Surg., 121(6):32932, 2001. 
BERTONE, V. H.; OTTONE, N. E.; LO TARTARO, M. A.; GARCÍA DE QUIRÓS, N.; ARROTEA, A.; DOMINGUEZ, M.; FINKELSTEIN, D.; LÓPEZ BONARDI, P.; SHINZATO, S. \& MEDAN, C. Consideraciones anátomo-clínicas sobre el nervio radial en el codo. Int. J. Morphol., 26(2):437-444, 2008.

Mazurek, M. T. \& Shin, A. Y. Upper extremity peripheral nerve anatomy: current concepts and applications. Clin. Orthop. Relat. Res., 383:7-20, 2001.

Monteiro, E.; Moura, A.; Barros, F.; Carvalho, P. \& Ferraro A. Lipoma causing a posterior interosseous nerve syndrome. Eur. J. Plast. Surg., 25:35-7, 2002.

Ozturk, A.; Kutlu, C.; Taskara, N.; Kale, A. C.; Bayraktar, B. \& Cecen, A. Anatomic and morphometric study of the arcade of Frohse in cadavers. Surg. Radiol. Anat., 27(3):171-5, 2005.

Riffaud, L.; Morandi, X.; Godey, B.; Brassier, G.; Guegan, Y.; Darnault, P. \& Scarabin, J. M. Anatomic bases for the compression and neurolysis of the deep branch of the radial nerve in the radial tunnel. Surg. Radiol. Anat., 21:229-33, 1999.

Roles, N. C. \& Maudsley, R. H. Radial tunnel syndrome: resistant tennis elbow as a nerve entrapment. J. Bone Joint Surg., 54(3):499-508,1972.

Roust, R. \& Espeche, R. C. División del nervio radial en el pliegue del codo. V Congreso de la Sociedad Rioplatense de Anatomía. Mendoza, 1969.

Sharrard, W. J. W. Posterior interosseous neuritis. J. Bone Joint Surg. Br., 48(4):777-80.1966.

Spinner, M. The arcade of Frohse and its relationship to posterior interosseous nerve paralysis. J. Bone Joint Surg. Br., 50(4):809-12, 1968.

Testut, L. \& Latarjet, A. Tratado de anatomía humana. 9a edición, : Angiología. Barcelona, Salvat, 1954. V. II.

Werner, C. O. Paralysis of the posterior interosseous nerve caused by tumour: brief report. J. Bone Joint Surg. Br., 69(4):670-1, 1987.

Witt, J. D. \& Kaminemi, S. The posterior interosseous nerve and the posterolateral approach to the proximal radius. J. Bone Joint Surg. Br., 80(2):240-2, 1998.

Younge, D. H. \& Moise, P. The radial tunnel syndrome. Int. Orthop., 18(5):268-70, 1994.
Dirección para correspondencia:

Nicolás E. Ottone

Melincué 5308

Código Postal: 1408

Ciudad Autónoma de Buenos Aires

ARGENTINA

Email: nicolasottone@gmail.com

Recibido : 30-09-2007

Aceptado: 09-04-2008 